\title{
An Empirical Study on the Relationship between Interaction Number and Marketing Effect of Movie Microblog
}

\author{
Siyi Wang ${ }^{*}$, Jie Long \\ School of Sichuan University, Chengdu, China \\ *Corresponding author: wsy17978@163.com \\ Received February 14, 2019; Revised March 24, 2019; Accepted April 06, 2019
}

\begin{abstract}
Nowadays microblog marketing has become a major marketing way for Chinese movies. On the basis of the AISAS theory model, a first-order autoregressive model between the interaction number and marketing effect of the movie's official microblog is constructed. Research shows that during the movie microblog marketing activities, there is a certain initial marketing effect, and interacting with fans has a certain amplification effect on the marketing effect, but eventually the marketing effect naturally declines with time.
\end{abstract}

Keywords: movie marketing, AISAS model, microblog interaction number, marketing effect

Cite This Article: Siyi Wang, and Jie Long, "An Empirical Study on the Relationship between Interaction Number and Marketing Effect of Movie Microblog.” Journal of Business and Management Sciences, vol. 7, no. x (2019): 59-63. doi: 10.12691/jbms-7-2-1.

\section{Introduction}

With the popularity of mobile terminals and the development of social media, more and more movie teams use microblog, which is the largest social platform in China, for marketing communication because of its unique communication advantages. However, the current study on film marketing effect evaluation of microblog is not enough, nor is there a clearer marketing plan for movie teams. At the same time, as a special cultural product, the marketing cycle of movies lasts generally only about two months. How to maximize the use of this marketing cycle to achieve the best marketing effect has become a concern of the film industry.

This study is based on the attention-interest-searchaction-share (AISAS) theory model, taking the movie The Wandering Earth as an example to do the empirical research, which was released in the Spring Festival in 2019. Establish a quantitative model to measure the influence of movie's official microblog interaction number on marketing effect, to study the effect and significance of the interaction between official microblog and fans on film marketing and communication, and propose corresponding management suggestions to the film team for the microblog marketing activities.

\section{Literature Review}

Microblogging marketing belongs to e-commerce and network marketing areas. [1] The related theory and literature had been developing worldwide in the past serveral years. Dentsu (2007) raised AISAS theory (Attention, Interest, Search, Action, Share) for network marketing (see Figure 1) [2], reflecting the process of influence attenuation and amplification effect of microblogging marketing. On this basis, research on the AISAS model has gradually deepened and the application field is expanding. Jin Yongsheng and Wang Rui believed that the short-term effect of enterprise microblog marketing is positively related to the number of microblog posts and the number of fans, and a short-term model for measuring the marketing effect of enterprise microblog is constructed according to the AISAS model. [3] Zeng Hongjiang regarded crowdfunding as an effective marketing approach, and builds an AR model based on AISAS marketing model and value co-creation theory to evaluate the marketing effect of crowdfunding. [4] Ge Yuhua combined with the AISAS model to analyze the whole process of microblog propagation of provincial satellite $\mathrm{TV}$, and constructed a quantitative model that can analyze the overall effect, influence and number of followers of microblog communication in provincial satellite TV. [5] Fu Yi qualitatively studied and analyzed the film social media marketing model based on the AISAS model, and proposed the marketing communication integration strategy for different stages of marketing. [6] Tirtha Dhar, Charles B. Weinberg explore the complexities of microblog interaction effects in non-linear models and outline methods to estimate and understand the movie interaction effects in two widely used marketing models. [7] Elif Ulker-Demirel investigates the impact of the importance, assigned by audiences, of factors such as people, movie features, script, price, promotion, and 
distribution channels (defined as a movie marketing mix) on the audience's buying intentions, as well as the impact of their buying intentions on word of mouth (WOM). [8]

The film is promoted and marketed through the microblog, mainly through the release of relevant posts to attract the attention of fans and stimulate their interests. Fans then search related information and purchase tickets on this basis, but the marketing influence will decay with time.

The official microblog of the movie will also continue to interact with fans and enhance the participation of fans, which will encourage fans to actively share film reviews and opinions, secondary communication and secondary creation. In this way, the microblog marketing effect of the movie will appear to be magnified.

Overall, film microblog marketing is basically matches with AISAS theory. The interaction number of official microblog (the number of posts been liked, commented and relayed) is a major factor to measure the effectiveness of film marketing in existing studies. Therefore, from the perspective of consumer behavior, this study considers the attenuation and amplification effect of microblog marketing influence. Taking the movie The Wandering Earth as an example, this study attempts to establish a first-order autoregressive model to explain the relationship between the official microblog interaction number and the marketing effect.

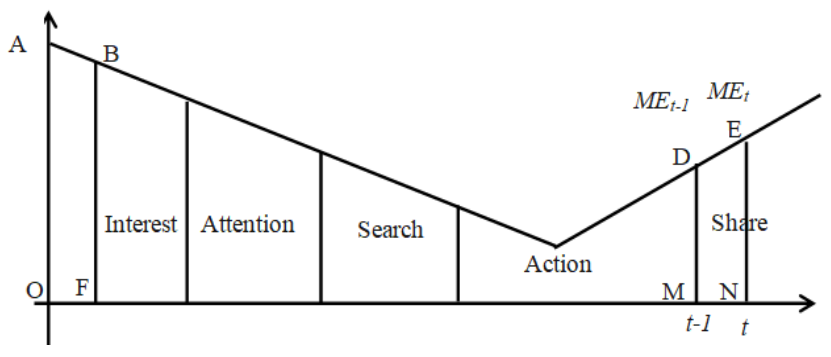

Figure 1. The AISAS Theory Model

\section{Model Building}

\subsection{Model Hypothesis}

Due to the popularity of the film itself and the actors, it has a certain influence in the early stage of marketing. At the same time, the film team will release information related to the film and interact with fans through the microblog platform, which will have a certain amplification effect on marketing effects. However, as a short-term cultural product, the effect of marketing through microblog will naturally decline over time. The number of movie keywords mentioned in the specified time range is used as an indicator to measure the marketing effect of the movie microblog. The total number of likes, comments and relays of the official microblog received can be regarded as an indicator to measure the interactive effect of the fans.

Based on the AISAS theory, draw a movie microblog marketing process figure (see Figure 1), in which the horizontal axis represents time, and the vertical axis represents marketing effect.

$O$ indicates the time origin, point $A$ indicates the initial influence of the film before the microblog marketing, which stems from the attention to the original novels or the actors. Point $B$ indicates the initial marketing effect of the film team on microblog when it started microblog marketing. The film attracts fans' attention by publishing relevant information and content elements, but its influence on the microblog platform will show a weakening trend as time goes by. In this natural decay process, the movie microblog has gone through an attention-interest step and earlier stage of search-actionshare step, point $C$ indicates that the influence of movie microblog naturally attenuate to the lowest point. Since then, with the release of the film and the increase of interaction with fans, the short-term effect of the movie microblog marketing has gradually emerged and formed a virtuous circle, and the influence gradually increases and enters the action-sharing stage. Take an observation timepoint $t$ in the share step, when the influence of movie microblog is $E$. $M E_{t}$ indicates the accumulated influence between time-point $O$ and $t$; then take an observation time-point $t-1$ before $t$, when the influence of movie microblog is $D, M E_{t-1}$ indicates the accumulated influence between time-point $O$ and $t-1 ; F, M, N$ are corresponding time-point of $B, D, E$ on time axis.

Variable definition used in model constructing based on AISAS theory is shown as follows:

- IN: The total number of interactions (the total numbers of likes, comments, and relays) that the movie microblog received

- $M E$ : The number of movie keywords mentioned by all microblog users

- $t$ : Any observation time-point

- $t-1$ : An observation time-point before time-point $t$

- MEt: The total number of the movie keyword mentioned by the microblog users between the time-point $O$ and the time-point $t$, which is used to measure the marketing effect produced by the microblog marketing between the time-point $O$ and the time-point $t$

- $M E_{t-1}$ : The total number of the movie keyword mentioned by the microblog users between the time-point $O$ and the time-point $t-1$, which is used to measure the marketing effect produced by the microblog marketing between the time-point $O$ and the time-point $t-1$

- $\triangle M E$ : Variable quantity of the marketing effect of movie microblog between time-point $t$ and timepoint $t-1$

- $a$ : The brand influence of the film before the microblog marketing

- $a_{0}$ : The marketing effect created during the process originated from $a$. It is a constant only related to $a$.

- $a_{1}$ : Natural attenuation coefficient of $M E_{t-1}, 0<a_{1}<1$

- $a_{2}$ : Amplification coefficient of the marketing effect of movie microblog from microblog interactive effect, $a_{2}>0$

\subsection{Model Establishment}

As Figure 1 shows, the original influence of the film on microblog marketing is $S_{A B F O}$. Taking time-point $t$ and $t-1$, and accumulated marketing effect of movie microblog between time-point $O$ and $t$ as $M E_{t}=S_{A E N O}$; accumulated marketing effect of movie microblog between time-point 
$O$ and $t-1$ as $M E_{t-1}=S_{A D M O}$, and during the observation period, variable quantity of the marketing effect of movie microblog is $\triangle M E=M E_{t}-M E_{t-1}=S_{D E N M}$.

Due to the complexity of multi-level propagation of microblog, the first-order autoregressive model is used to quantitatively study the influencing factors of $\triangle M E$, which is mainly affected by the initial marketing effect, the official microblog interaction number $I N_{t}$ and the previous time-point marketing effect $M E_{t-1}$. Since the number of the keyword mentioned and the interaction number of the microblog are very large, they are processed logarithmically.

Based on this, the following first-order autoregressive model is established:

$$
\ln M E_{t}=a_{0}+a_{1} \ln M E_{t-1}+a_{2} \ln I N_{t} .
$$

Among them, $a_{0}$ indicates the initial marketing effect of the movie; $a_{1}$ indicates the influence degree on current marketing effect which affected by the previous marketing effect; $a_{2}$ indicates the impact of the official microblog interaction number on the marketing effect in the short-term; $t$ indicates time.

\section{Empirical Research}

\subsection{Data Source}

Taking The Wandering Earth as an example, we crawled the data of the film's keywords mentioned through the crawler software, the number of microblogs posted by the official microblog, including the content, the number of likes, comments and relays in every day from January $11^{\text {th }}, 2019$ to March $5^{\text {th }}, 2019$ (from the beginning of pre-sale to the end of film show), totaling $T=54$ sets of time series data.

\subsection{Descriptive Statistics}

For these 54 sets of time series data, a total of $N=324$ sets of valid panel data are used to make descriptive statistics. The results are shown in Table 1. Draw the results of the number of keywords mentioned of each set of time series data and the logarithm of the total interaction number over time, as is shown in Figure 2. It can be seen that after the pre-sale began, the film team began to enter the stage of marketing momentum. In the early stage, the number of keywords mentioned and interactions soared simultaneously and reached the peak through the detonation of hot events. Thereafter, the popularity decreases, both of them showed a downtrend, but with the release of the film, it rose again. And eventually showed a natural decay trend over time.

Table 1. Descriptive Statistics of Data

\begin{tabular}{|c|c|c|c|c|c|}
\hline & $\begin{array}{c}\text { Number of } \\
\text { Keywords } \\
\text { Mentioned }\end{array}$ & likes & comments & relays & $\begin{array}{c}\text { Interaction } \\
\text { number }\end{array}$ \\
\hline average & 237,844 & 11,536 & 3,047 & 47,019 & 61,602 \\
\hline maximum & $1,735,623$ & 68,454 & 22,692 & $1,252,311$ & $1,292,790$ \\
\hline minimum & 8,216 & 627 & 177 & 82 & 969 \\
\hline
\end{tabular}

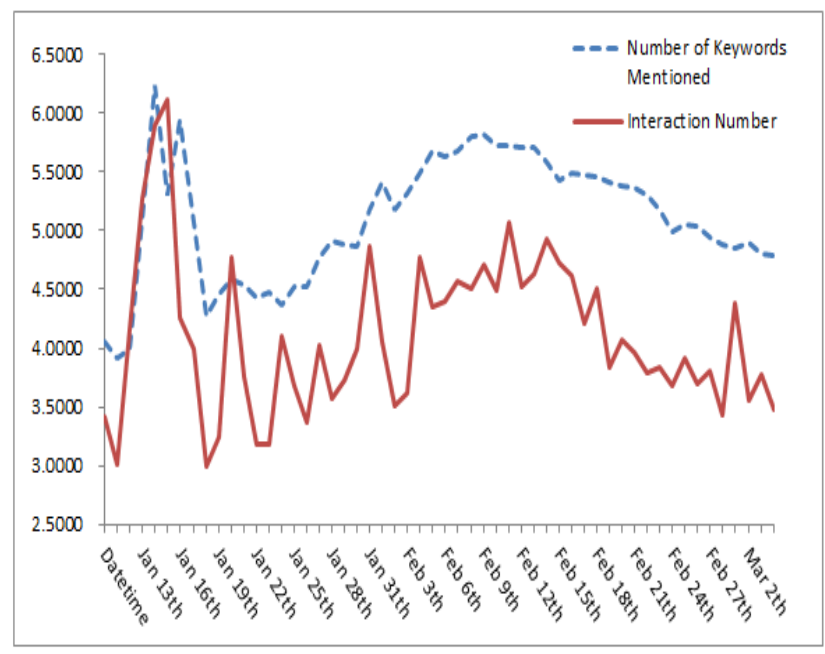

Figure 2. Dynamic Changes of the Number of Keywords Mentioned and Interaction Number

\subsection{Unit Root Test}

In order to avoid pseudo-regression caused by non-stationary time series, the panel data of the number of keywords mentioned and interaction number are logarithmically processed, and the stationarity of the panel data is analyzed by unit root test to ensure the validity of the estimated results. Panel data $N=324, T=54$. In Eviews software, ADF method is used for unit root test. The test results are shown in Table 2.

Table 2. Unit Root Test

\begin{tabular}{|c|c|c|c|}
\hline variable & T statistics & probability & results \\
\hline $\ln M E_{t}$ & $-15.3162^{* * *}$ & 0.0000 & stable \\
$\ln I N_{t}$ & $-5.8924^{* * *}$ & 0.0001 & stable \\
\hline
\end{tabular}

Notation: *** indicates that the level above $1 \%$ is significant.

The results show that the original assumption of unit root is rejected at $1 \%$ significance level. The above variables are stationary series.

\subsection{Empirical Results and Discussions}

According to formula (1), 54 sets of valid data of Wandering Earth are fitted and regressed by Eviews software. The results are shown in Table 3.

Table 3. Fitting Regression of Microblog Marketing Effect

\begin{tabular}{|l|c|c|c|}
\hline variable & coefficient & T statistics & probability \\
\hline $\ln M E_{t-1}$ & 0.6011 & $10.7321^{* * *}$ & 0.0000 \\
\hline $\ln I N_{t}$ & 0.3217 & $4.4457^{* * *}$ & 0.0000 \\
\hline$a_{0}$ & 1.5212 & $3.2594^{* * *}$ & 0.0020 \\
\hline Observations: 54 & \multicolumn{2}{|c|}{$R^{2}=0.959$} \\
\hline
\end{tabular}

Notation: *** indicates that the level above $1 \%$ is significant.

(1) $R_{2}=0.959$, indicating that the fitting degree of model is very high.

(2) $a_{0}=1.5212>0$, showing that the film has a certain initial marketing effect in the early stage of marketing, which stems from the influence of the original novel and the popularity of actors. 
Table 4. Fitting Regression of Marketing Effect at Different Stages

\begin{tabular}{|c|c|c|c|c|c|c|c|c|c|}
\hline \multirow{2}{*}{ coefficient } & \multicolumn{3}{|c|}{ before the film is released } & \multicolumn{3}{c|}{ mid-release of the film } & \multicolumn{3}{c|}{ later stage of film release } \\
\cline { 2 - 10 } & coefficient & T-statistic & probability & coefficient & T-statistic & probability & coefficient & T-statistics & probability \\
\hline a1 & 0.241 & $3.342^{* * *}$ & 0.003 & 1.069 & $22.005^{* * *}$ & 0.000 & 0.837 & $40.913^{* * *}$ & 0.000 \\
\hline a2 & 0.639 & $8.170^{* * *}$ & 0.000 & 0.423 & $5.189^{* * *}$ & 0.011 & 0.165 & $1.514^{*}$ & 0.055 \\
\hline a0 & 2.044 & $4.966^{* * *}$ & 0.000 & 0.237 & $3.845^{* * *}$ & 0.004 & 0.180 & $1.360^{*}$ & 0.089 \\
\hline
\end{tabular}

Notation: *, **, *** indicate significant levels above $10 \%, 5 \%$ and $1 \%$ respectively.

(3) $a_{1}>0$, indicating that the current marketing effect of the movie is affected by the previous marketing effect and positively correlated; $0<a_{1}<1$, indicating that the marketing effect is a natural decline trend. $a_{2}>0$, indicates that the interaction between official microblog and fans has a certain magnification effect on film marketing. The higher the interaction degree, the more obvious the marketing effect.

Different marketing stages have different marketing effects, and the interaction number of microblog has different magnifying effect on marketing effect. According to AISAS model, the participating process of consumers in marketing activities can be divided into five stages: attention-interest-search-action-sharing. This paper divides the marketing process of The Wandering Earth into three stages: Before the release of the film (Jan $1^{\text {th }}-$ Feb $4^{\text {th }}$, arousing attention and interest), in the middle of the release (Feb $5^{\text {th }}$-Feb $17^{\text {th }}$, information search and tickets purchase), and in the later stage (Feb $18^{\text {th }}$-Mar $5^{\text {th }}$, film reviews and opinions sharing). The effects of different stages of film marketing are analyzed, and the results are shown in Table 4.

(1) $a_{0}$ is getting smaller and smaller, which indicates that the initial marketing effect decreases with time.

(2) $0<a_{1}<1$, indicating that the current marketing effect is positively correlated with the previous marketing effect at all stages of marketing, and the marketing effect is declining. At the same time, $a_{1}$ is increasing, indicating that the decay of marketing effect is more obvious in the early stage. In the medium-term the coefficient is greater than 1, indicating that enterprises have other marketing channels besides official microblog marketing, such as the whole network attention and search caused by the release of movies, the transfer of opinion leaders and so on. Later $a_{1}$ declines again, but still close to 1 , indicating that the trend of attenuation is gradually decreasing, and the current marketing effect basically depends on the marketing effect of the previous period.

(3) $a_{2}$ is bigger in the early and middle stages, and there are obvious attenuation in the later stages. This shows that the magnification effect of the interaction exists in three stages, but it is more obvious in the early and middle stages, and smaller in the later stages. Because the cycle of the film is short, so the marketing effect shows a significant attenuation trend in the later stage of the film release.

\section{Conclusions}

Based on AISAS model, this paper constructs a first-order autoregressive model for interaction number and marketing effect of movie official microblog. Through empirical analysis, the following conclusions are drawn:
(1) The official microblog of the film should strengthen the interaction with fans. According to the model, it is verified that the interaction number with fans can obviously magnify the marketing effect of the film. Good interaction effect will further increase fans' interest and participation, thus stimulating them to purchase tickets and share opinions.

(2) Seize the opportunity before the release of the film to promote and interact. It can be seen from the parameters after model fitting regression that the amplification effect of interaction number on marketing effect is most obvious before and at the beginning of the release of the film, especially during the period before the release of the film, which is the best time for promotion. Positive interaction with fans in this stage can effectively improve the attention and expectations of fans.

(3) Selecting the proper stars for propagandizing will have an obvious effect in a short period of time. In the early stage of marketing, The Wandering Earth attracted the attention of the whole Internet through hot events. It was because the official microblog released the $M V$ of theme song by Meng Meiqi, which received 7 million relays.

(4) Pay attention to long-term maintenance of the brand. After the movie end releasing, most of the official microblogs stopped updating information immediately, leading to the accumulated fans and attention lost their values. Therefore, the film team should pay attention to the building and maintenance of the film brand. In the later stage or after the film ends releasing, they should actively promote the peripheral products of the film. Keep active on microblog and carry out regular marketing activities at the same time, so as to make the film brand truly lasts popular.

From the perspective of marketing effect evaluation, this study constructs the AR model of film microblog interaction number and marketing effect, and further analyzes the marketing effect at different stages. The research results have certain reference value for film teams in microblog marketing and communication.

The paper still has certain deficiencies. For example, use the number of keywords mentioned to measure marketing effect has a certain error, for which we don't list all the keywords related to the movie. Meanwhile, which type of microblog can have a crucial effect to the interaction number has not been discussed. This will be discussed in the future.

\section{References}

[1] Wang Rui, Jin Yongsheng. An Empirical Study on the Relationship between the Followers' Number and Influence of 
Microblogging[P]. E-Business and E-Government (ICEE), 2010 International Conference on, 2010.

[2] Dentsu, "AISAS reconstructs the model of consumer's behavior in network era," Modern Advertisement, vol. 89, pp. 23-24, Feb. 2007 (In Chinese).

[3] Jin Yongsheng, Wang Rui, Chen Xiangbing. Short-term Interactive Model of Enterprise Microblog Marketing Effect and Number of Followers [J]. Management Science, 2011, 24(04): 71-83.

[4] Zeng Jianghong, Huang Xiangrong, Chen Ye. Research on Dynamic Marketing Effect of Crowdfunding Projects Based on AISAS Behavior Model [J]. Journal of central south university (social science edition), 2019, 25(01): 113-120.
[5] Ge Weihua. Analysis of Micro-blog Communication Effect of Provincial TV Stations Based on AISAS Model [J]. Media, 2016(11): 89-91.

[6] Fu Yi. Research on the Construction of Film Social Media Marketing Model Based on AISAS Model (ii)[J]. Chinese Film Market, 2018(12): 22-24.

[7] Tirtha Dhar, Charles B. Weinberg. Measurement of interactions in non-linear marketing models: The effect of critics' ratings and consumer sentiment on movie demand[J]. International Journal of Research in Marketing, 2016, 33(2).

[8] Elif Ulker-Demirel, Ayse Akyol, Gülhayat Gölbasi Simsek. Marketing and consumption of art products: the movie industry[J]. Arts and the Market, 2018, 8(1).

(C) The Author(s) 2019. This article is an open access article distributed under the terms and conditions of the Creative Commons Attribution (CC BY) license (http://creativecommons.org/licenses/by/4.0/). 